\title{
How the three arches of the foot intercorrelate
}

\author{
A.S. Gwani ${ }^{1}{ }^{2}$, M.A. Asari ${ }^{1}$, Z.I. Mohd Ismail ${ }^{1}$ \\ ${ }^{1}$ Department of Anatomy, School of Medical Sciences, Universiti Sains Malaysia, Kampus Kesihatan, Kelantan, Malaysia \\ 2Department of Human Anatomy, College of Medical Sciences, Abubakar Tafawa Balewa University, Bauchi State, Nigeria \\ [Received: 13 March 2017; Accepted: 1 May 2017]
}

Background: The foot is composed of medial, lateral and transverse arches which, particularly the medial arch, provide it with the ability to function both as a flexible and rigid structure for proper locomotion. Arches of the foot, as well as their effect on lower extremity function, have been studied. However, quantitative data on the relationship between these arches still remain scanty. The purpose of this study was, therefore, to examine how the three arches of the foot intercorrelate. Materials and methods: Seventy-six participants (58 males, 18 females) were recruited to participate in the study. Bilateral weight-bearing lateral radiographs of the right foot were taken from each participant. Navicular heights (NH), medial cuneiform height $(\mathrm{MCH})$, calcaneal inclination angle (CIA) and calcaneal-first metatarsal angle (C1MA) were measured to represent the medial arch. The lateral arch was represented by cuboid height $(\mathrm{CH})$ and calcaneal-fifth metatarsal angle (C5MA) whereas; $\mathrm{MCH}$ and $\mathrm{CH}$ represented the transverse arch. Mean difference of variables between males and females was compared using independent $t$-test while the correlation between the variables was determined using Pearson correlation.

Results: All the variables were not significantly related to gender. Significant moderate to excellent linear correlations were observed between the variables. CIA showed the strongest correlation with C1MA $(r=-0.90)$ and C5MA $(r=-0.84)$ whereas, $\mathrm{CH}$ had the least correlation with other variables.

Conclusions: The moderate to excellent correlations between the variables indicate that deformation or elevation of the medial arch may consequently result in similar movements of the lateral and transverse arches and vice versa. (Folia Morphol 2017; 76, 4: 682-688)

Key words: foot, arch, correlation, morphology, deformity

\section{INTRODUCTION}

The human foot is a complex anatomical and biomechanical structure. Obviously, due to the complex interaction between the anatomical structures it comprises, the foot is able to provide a stable base to support standing posture as well as change from flexible structure that dissipates impact as it strikes the ground and accepts the body's weight to a rigid structure that allows efficient propulsion during toeoff $[11,26]$.

Bones of the foot are arranged in a longitudinal and transverse manner, forming a dome relative to the ground, which gives rise to longitudinal and transverse arches on the plantar aspect of the 
foot [9]. The longitudinal arch consists of medial arch on the medial side and a lateral arch on the lateral side of the foot. The medial arch is made up of calcaneus, talus, navicular, the three cuneiforms and first three metatarsals. The arch is higher above the ground compared to the lateral and transverse arches due to the orientation of its skeletal components. The lateral arch, on the other hand, is made up of the calcaneus, cuboid, and lateral two metatarsals. It is lower than the medial arch and lies in close contact with the ground in weight-bearing foot. Nevertheless, it plays a vital role in providing support as the body's weight is distributed to both medial and lateral arches during locomotion [29, 30]. The transverse arch traverses from a higher medial to lower lateral side of the tarsometatarsal region of the foot. It is made up of the three cuneiforms and cuboid proximally and bases of the five metatarsals distally.

Morphology of the medial arch is often used to categorise foot type into three: pes rectus, pes planus, and pes cavus. Pes rectus is a normal (normally aligned) foot. The arch height is within normal range and bisector of the posterior surface of the calcaneus is perpendicular to the ground. The arch height of pes planus (flatfoot) is below the normal range and the plantar aspect of the foot is in complete or near-complete contact with the ground. It is also characterised by everted calcaneus. Pes cavus is characterised by an abnormally high-arched foot with an inverted calcaneus. Evidently, the medial arch is the main point of reference in diagnosis of pes planus or pes cavus [32], although the morphology of both lateral and transverse arches may also provide valuable information in the diagnosis of these deformities.

While it has been clinically explained that the medial and lateral arches function as a unit with the transverse arch $[1,17]$, there is scarcity of data to quantify this relationship. Indeed, previous studies were mainly limited to the medial arch, while in reality; all the three arches contribute to support the diverse biomechanical activities of the foot. Moreover, it has been shown that motion of the lateral arch is similar to that of the medial arch $[9,14,19$, 20]; whereas the transverse arch is essential to the stability of both arches $[2,22]$. Thus, understanding the relationship between the arches of the foot is paramount. The objective of this study, therefore, was to examine how the three arches of the foot intercorrelate.

\section{MATERIALS AND METHODS}

\section{Participants}

Seventy-six individuals (58 males, 18 females) aged 18-35 years volunteered to participate in the study. The participants were randomly selected from patients attending Radiology Department of the Abubakar Tafawa Balewa University Teaching Hospital (ATBUTH), Bauchi, Nigeria. Their consent was sought as they attend the department for various radiological procedures. These participants had no history of lower extremity deformity (except flatfoot or high-arched foot). The study was carried out after receiving approval from Human Ethics Committee of the ATBUTH.

\section{Procedures}

Lateral radiographs of the right foot of each participant were taken. The participants were asked to stand on a custom-built wooden platform $25 \mathrm{~cm}$ high, keeping their feet beside two straight lines drawn $15 \mathrm{~cm}$ apart on the platform $[4,23]$. They were also instructed to place equal weight on both feet. The radiographic film cassette was placed vertically adjacent to the medial border of the right foot. A longitudinal cut was made on the platform to guide placement of the cassette so that its lower edge can descend some distance inferior to the plantar aspect of the foot. The X-ray tube was kept at a distance of $100 \mathrm{~cm}$ from the cassette and the beam was centered horizontally at the lateral malleolus. The same exposure of $6.3 \mathrm{mAs}$ at $55 \mathrm{KV}$ was maintained for each participant while acquiring the radiographs. All radiographs were taken by the same radiographer.

\section{Measurements}

All measurements were made directly on the radiographs. Each radiograph was placed on X-ray viewer and a sharp clutch pencil was used to draw lines on the anatomical landmarks required for the measurements. Height (Fig. 1) and angles (Fig. 2) were measured using a plastic ruler and a universal plastic goniometre respectively. Navicular height $(\mathrm{NH})$ was defined as the perpendicular distance from the most inferior aspect of the navicular bone to the horizontal supporting surface [15]. Medial cuneiform height $(\mathrm{MCH})$ was measured as the perpendicular distance from the most inferior aspect of the medial cuneiform bone to the horizontal supporting surface [15]. Calcaneal inclination angle (CIA) was 


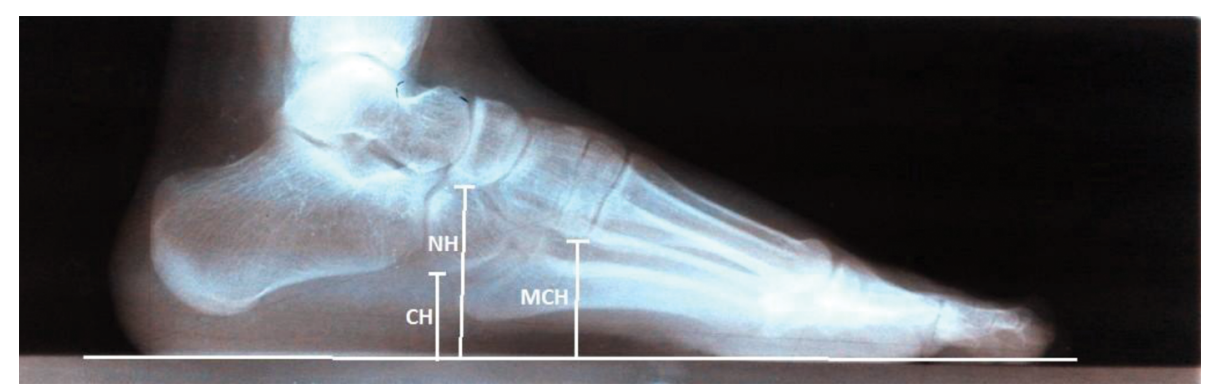

Figure 1. Measurement of navicular height (NH), cuboid height $(\mathrm{CH})$ and medial cuneiform height $(\mathrm{MCH})$.

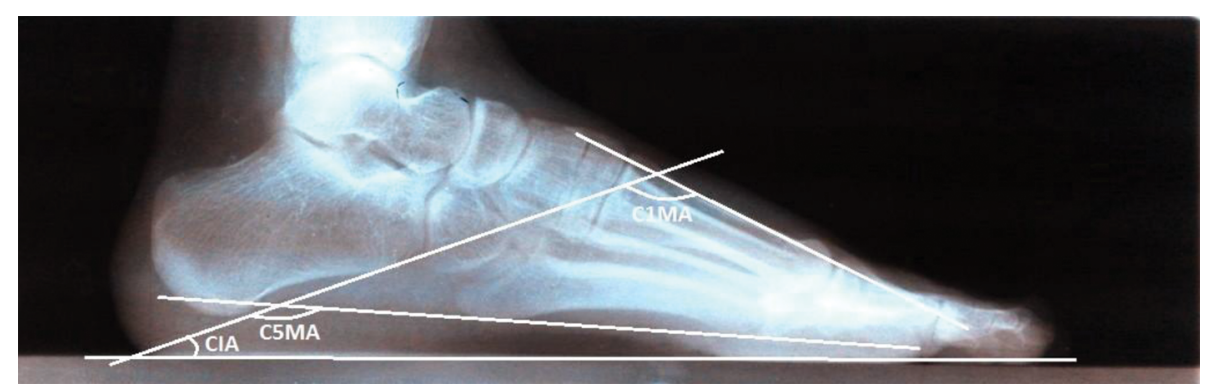

Figure 2. Measurement of calcaneal inclination angle (CIA), calcaneal-first metatarsal angle (C1MA) and calcaneal-fifth metatarsal angle (C5MA).

measured as the angle formed between the tangent to the inferior aspect of the calcaneus and horizontal supporting surface [25]. Calcaneal-first metatarsal angle (C1MA) was measured as the angle formed between the tangent to the inferior surface of the calcaneus and a line drawn along the dorsum of the midshaft of the first metatarsal [18]. Cuboid height $(\mathrm{CH})$ was measured as the perpendicular distance from the most inferior aspect of the cuboid to the horizontal supporting surface [15]. The calcanealfifth metatarsal angle (C5MA) was defined as the angle formed between the tangent to the inferior aspect of the calcaneus and a line drawn along the inferior aspect of the base and head of fifth metatarsal [15].

\section{Statistical analysis}

All statistical analyses were performed using IBM SPSS Statistics (version 22) software, Armonk, New York. The descriptive statistics of the variables measured were explored. Kolmogorov-Smirnov test was used to check whether the data were normally distributed before conducting further analysis. The level of significance in all statistical analyses was set at $p<0.05$. Independent $t$-tests were used to determine whether mean differences exist between males and females in the measured variables. Homogeneity of variances was checked using Levene's test. Since no significant difference was obtained, the data were pooled in the subsequent analyses. A simple scatter plot showed a bivariate normal distribution between all variables. Strength of the relationship between the variables was determined using Pearson's correlation and the results were interpreted based on the guidelines of [5]: little/no correlation (0.00-0.24), fair correlation (0.25-0.49), moderate/good correlation (0.50-0.74), and good/excellent correlation (0.75-1.00).

\section{RESULTS}

The descriptive statistics of the variables studied are presented in Table 1.

The results of the independent $t$-tests (presented in Table 2) indicates no significant difference between males and females for any of the variables ( $p>0.05$ and $95 \%$ confidence interval crosses zero in all variables).

The results of the Pearson correlations showed moderate to excellent correlations between the respective variables representing the three arches of the foot (Table 3). The C1MA and C5MA were positively correlated, whereas their correlation with other vari- 
Table 1. Descriptive statistics of the studied variables $(n=76)$

\begin{tabular}{lccccc}
\hline Variables & Gender & Mean (SD) & 95\% Cl & Minimum & Maximum \\
\hline $\mathrm{NH}[\mathrm{cm}]$ & Combined & $2.69(0.68)$ & $2.53-2.86$ & 0.70 & 4.20 \\
& Male & $2.74(0.68)$ & $2.56-2.92$ & 0.70 & 4.20 \\
& Female & $2.52(0.69)$ & $2.18-2.86$ & 1.00 & 3.80 \\
$\mathrm{MCH}[\mathrm{cm}]$ & Combined & $1.97(0.51)$ & $1.86-2.09$ & 0.70 & 3.00 \\
& Male & $2.03(0.48)$ & $1.91-2.16$ & 0.70 & 3.00 \\
& Female & $1.78(0.56)$ & $1.50-2.05$ & 0.70 & 2.80 \\
$\mathrm{CIA}\left[^{\circ}\right]$ & Combined & $14.67(4.70)$ & $13.60-15.75$ & 4.00 & 26.00 \\
& Male & $14.57(4.42)$ & $13.41-15.73$ & 5.00 & 26.00 \\
$\mathrm{C1MA}\left[{ }^{\circ}\right]$ & Female & $15.00(5.64)$ & $12.20-17.80$ & 4.00 & 21.00 \\
& Combined & $142.88(7.63)$ & $141.14-144.63$ & 122.00 & 162.00 \\
& Male & $142.83(7.39)$ & $140.88-144.77$ & 122.00 & 158.00 \\
$\mathrm{CH}[\mathrm{cm}]$ & Female & $143.06(8.60)$ & $138.78-147.33$ & 135.00 & 162.00 \\
& Combined & $1.12(0.33)$ & $1.04-1.20$ & 0.40 & 1.90 \\
$\mathrm{C5MA}\left[{ }^{\circ}\right]$ & Male & $1.12(0.33)$ & $1.03-1.21$ & 0.60 & 1.90 \\
& Female & $1.12(0.34)$ & $0.95-1.30$ & 0.40 & 1.80 \\
& Combined & $162.75(5.58)$ & $161.47-164.03$ & 151.00 & 176.00 \\
\hline
\end{tabular}

$\mathrm{NH}$ — navicular height; $\mathrm{MCH}$ - medial cuneiform height; $\mathrm{ClA}$ — calcaneal inclination angle; $\mathrm{C} 1 \mathrm{MA}$ — calcaneal-first metatarsal angle; $\mathrm{CH}$ - cuboid height; $\mathrm{C} 5 \mathrm{MA}$ — calcaneal-fifth metatarsal angle; $\mathrm{Cl}$ - confidence interval; SD — standard deviation

Table 2. Result of independent t-test between males and females

\begin{tabular}{lccc}
\hline Variables & $\begin{array}{c}\text { Mean diff. } \\
(95 \% \text { Cl) }\end{array}$ & $\begin{array}{c}\text { t-statistics } \\
\text { (df) }\end{array}$ & $\mathbf{P *}^{*}$ \\
\hline $\mathrm{NH}[\mathrm{cm}]$ & $0.23(-0.14,0.59)$ & $1.32(74)$ & 0.222 \\
$\mathrm{MCH}[\mathrm{cm}]$ & $0.26(-0.01,0.53)$ & $1.90(74)$ & 0.061 \\
$\mathrm{CIA}\left[^{\circ}\right]$ & $-0.43(-2.97,2.11)$ & $-0.34(74)$ & 0.736 \\
$\mathrm{C} 1 \mathrm{MA}\left[{ }^{\circ}\right]$ & $-0.23(-4.36,3.90)$ & $-0.11(74)$ & 0.913 \\
$\mathrm{CH}[\mathrm{cm}]$ & $-0.00(-0.18,0.18)$ & $-0.04(74)$ & 0.971 \\
$\mathrm{C} 5 \mathrm{MA}\left[{ }^{0}\right]$ & $1.06(-1.96,4.07)$ & $0.70(74)$ & 0.487 \\
\hline
\end{tabular}

${ }^{*} p$ - no significant gender difference in all variables using independent t-test $(p>0.05)$; $\mathrm{NH}$ - navicular height; $\mathrm{MCH}$ - medial cuneiform height; $\mathrm{CIA}$ - calcaneal inclination angle; $\mathrm{C} 1 \mathrm{MA}$ - calcaneal-first metatarsal angle; $\mathrm{CH}$ — cuboid height; C5MA — calcaneal-fifth metatarsal angle; $\mathrm{Cl}$ - confidence interval ables was negative. The correlation coefficient ranged from 0.53 to 0.84 for positive correlations, and from -0.51 to -0.90 for negative correlations ( $p<0.001$ in all the correlation analyses).

\section{DISCUSSION}

The medial arch is the chief load-bearing structure in the foot and clinically the most important of the three arches of the foot [10]. NH is proportional to the height of the arch and this makes the navicular bone a suitable reference point in examinations of arch deformities [24]. The present study obtained good correlation between this variable and other measures of medial arch morphology (Table 3). The

Table 3. Correlation between the variables

\begin{tabular}{|c|c|c|c|c|c|c|}
\hline Variables & C5MA [0] & $\mathrm{CH}[\mathrm{cm}]$ & C1MA [0] & CIA [ [0] & $\mathrm{MCH}[\mathrm{cm}]$ & $\mathrm{NH}[\mathrm{cm}]$ \\
\hline $\mathrm{NH}[\mathrm{cm}]$ & -0.56 & 0.53 & -0.77 & 0.71 & 0.77 & 1 \\
\hline $\mathrm{MCH}[\mathrm{cm}]$ & -0.51 & 0.56 & -0.72 & 0.66 & 1 & \\
\hline CIA [0] & -0.84 & 0.54 & -0.90 & 1 & & \\
\hline C1MA [0] & 0.84 & -0.59 & 1 & & & \\
\hline $\mathrm{CH}[\mathrm{cm}]$ & -0.53 & 1 & & & & \\
\hline C5MA [0] & 1 & & & & & \\
\hline
\end{tabular}

All variable showed strong significant linear correlation ( $<$ < 0.001); $\mathrm{NH}$ - navicular height; $\mathrm{MCH}$ - medial cuneiform height; CIA — calcaneal inclination angle; C1MA — calcaneal-first metatarsal angle; $\mathrm{CH}$ - cuboid height; C5MA — calcaneal-fifth metatarsal angle 
correlation coefficients were $0.77,0.71$, and -0.77 with $\mathrm{MCH}, \mathrm{CIA}$, and $\mathrm{C} 1 \mathrm{MA}$, respectively. Thus, as the height of the medial arch decreases, the $\mathrm{NH}, \mathrm{MCH}$, and $\mathrm{CIA}$ also decrease whereas the C1MA increases. Indeed, these variables are important parameters in the examination of the morphology of the medial arch $[15,16,25]$.

On the other hand, it has been previously reported that calcaneocuboid joint and fifth tarsometatarsal joints are associated with the height of the lateral arch [29]. Hence, $\mathrm{CH}$ and C5MA could be important indicators of lateral arch morphology. Indeed, both variables have been used to represent the lateral arch as well as to classify foot type $[9,15]$. This study found a moderate negative correlation between these variables $(r=-0.53)$. Thus, flattening of the lateral arch reduces the $\mathrm{CH}$ which in turn increases the C5MA.

Moderate to excellent correlation between the skeletal components of the medial and lateral arches has been observed in the current study (Table 3). This finding is in agreement with Lautzenheiser and Kramer [13] who reported a good correlation between medial and lateral arches $(r=0.79)$. Additionally, the CIA has the highest correlation coefficient with C1MA $(r=-0.90)$ and C5MA $(r=-0.84)$ in our study, which indicates its relative importance to the aches. This finding is also supported by the findings of Lautzenheiser and Kramer [13]. The authors reported an excellent correlation between this angle and medial arch $(r=-0.81)$ as well as the lateral arch $(r=-0.94)$ [13]. This excellent correlation could be attributed to the fact that calcaneus is common to both arches. Therefore, its inclination (measured as $\mathrm{CIA}$ ) is important to their morphology. Furthermore, all the three angles reflect the sagittal plane alignment of the hindfoot and forefoot [18].

The correlation obtained between the measures of the medial and lateral arches could be better understood by considering the motions and relationship between the Chopart's joint and subtalar joint complex. The former joint lies in a plane transverse to the medial and lateral arches while the latter joint plays the role of the keystone during movements by distributing gravitational forces to the arches [29]. This relationship is as a result of the dual membership of the talonavicular joint. Firstly, the joint is part of the subtalar joint complex and secondly, it is anatomically and functionally linked to the calcaneocuboid joint to make up the Chopart's joint [27]. In addition, the longitudinal axes of Chopart's joint are similar to the longitudinal component of the subtalar joint axis, allowing inversion and adduction or eversion and abduction of the forefoot whereas, their oblique axes are similar to the axis of the ankle joint and provide mainly plantar flexion and dorsiflexion $[6,21]$. Thus, these joints exhibit simultaneous complex movements that are associated with inversion and eversion of the foot. Inversion consists of plantar flexion, adduction, and supination of the subtalar joints and adductionsupination of the navicular and cuboid (respective keystones of medial and lateral arches). Eversion on the other hand, consists of dorsiflexion, abduction, and pronation of the navicular and cuboid [30]. Indeed, Kapandji [12] previously reported that both the navicular and cuboid glide inferiorly during supination and vice versa during pronation. Specifically, the navicular glides medially and inferiorly on the talar head, whereas the cuboid follows the navicular, moving medially and inferiorly on the calcaneus during supination. Conversely, these relative movements are reversed during pronation. Therefore, the navicular and cuboid consistently move as a functional unit at the same time and in the same direction relative to the calcaneus and talus [20].

In view of the above, it can be clearly understood that deformation or elevation of the medial arch may lead to similar motion on the lateral arch. For instance, deformation of the medial arch as seen in pes planus deformities may result in the collapse of the lateral arch, except in rare cases such as pes planocavus deformity, a type of pes planovalgus deformity characterised by sagging of the medial arch and cavus deformity of the lateral arch [3]. On the other hand, abnormal elevation of medial arch such as in pes cavus deformities also elevates the lateral arch. The apex of both medial and lateral arches is not in contact with the ground regardless of whether the deformity is anterior, posterior or mixed [31].

Clinically, the region of the transverse arch consists of complex skeletal and capsuloligamentous structures that provide stability to the midfoot and forefoot. Injury to this region involves at least some disruption of the transverse arch [7], which subsequently flattens the medial and lateral arches $[2,22]$. The complications of this injury commonly result to midfoot instability and planovalgus deformity, and less commonly the cavus deformity [22]. Indeed, a study among subjects with diabetes mellitus and peripheral neuropathy associated with Charcot neuroarthropathic midfoot deformity reveals limited 
ankle joint plantar flexion, reduced inversion of the subtalar joint and decrease in CIA [28]. Consequently, these changes result in flattening of the medial and lateral arches.

Despite its significance to the stability of both medial and lateral arches, the transverse arch receives the least attention in the literature. It has been reported that the configuration of the three cuneiforms, cuboid and metatarsal bases form buttress for the three arches [7]. Through this configuration, the medial cuneiform turns out to be a common skeletal component to the medial and transverse arches, while the cuboid is common to the lateral and transverse arches. The variables representing the transverse arch in this study were the $\mathrm{MCH}$ and $\mathrm{CH}$. The correlation coefficient between these variables was found to be good in this study $(r=0.56)$. Good correlations have also been observed between these variables and other measures of the medial and lateral arches (Table 3). Indeed, the morphology of the three arches can be significantly determined from the measurement of these bones. Furthermore, it is known that through the windlass mechanism, the plantar fascia coils around the metatarsal heads when the metatarsophalangeal joints are dorsiflexed, pulling the calcaneus and metatarsal heads closer. This reduces calcaneal eversion and increases midfoot and forefoot pronation [27]. Consequently, all the three arches become elevated. Conversely, according to Franco [8], pronation of the forefoot, which flattens the medial arch, also causes flattening of the transverse arch by splaying the metatarsals. Thus, considering the findings of this study, the relationship between the three arches of the foot should be considered during the diagnosis and management of the deformities associated with arches of the foot. Similarly, this relationship should be taken into account when designing foot orthoses for the management of arch deformities.

\section{Limitations of the study}

There are some limitations to our study. Firstly, this study was carried out under static conditions which cannot represent the overall dynamic behaviour of the arches. Secondly, measurements were conducted on lateral radiographs alone, which are two-dimensional images. Thus, it is not possible to study the entire skeletal components of the arches. In particular, we did not include the keystone of the transverse arch (i.e. either of the intermediate cuneiform or the base of the second metatarsal as it has been debated).
Therefore, further three-dimensional in vivo studies are required to understand the relationship of the entire skeletal component as well as the morphological relationships of the arches during gait.

\section{CONCLUSIONS}

The present study has provided a quantitative data on the morphological relationship between the three arches of the foot. By implication, it may be understood that elevation or deformation of the medial arch, for instance, may generally bring about similar movements of the lateral and transverse arches and vice versa.

\section{REFERENCES}

1. Akdoğan I, Akkaya S, Akkaya N, et al. Comparison of the calcaneal pitch angle and modified projection area per length squared method for medial longitudinal arch evaluation of the foot. Balkan Med J. 2012; 29(4): 406-409, doi: 10.5152/balkanmedj.2012.036, indexed in Pubmed: 25207043.

2. Bálint GP, Korda J, Hangody L, et al. Regional musculoskeletal conditions: foot and ankle disorders. Best Pract Res Clin Rheumatol. 2003; 17(1): 87-111, doi: 10.1053/ yberh.2003.275, indexed in Pubmed: 12659823.

3. Bourdet $C$, Seringe $R$, Adamsbaum $C$, et al. Flatfoot in children and adolescents. Analysis of imaging findings and therapeutic implications. Orthop Traumatol Surg Res. 2013; 99(1): 80-87, doi: 10.1016/j.otsr.2012.10.008, indexed in Pubmed: 23260367.

4. Buldt AK, Murley GS, Levinger P, et al. Are clinical measures of foot posture and mobility associated with foot kinematics when walking? J Foot Ankle Res. 2015; 8: 63, doi: 10.1186/s13047-015-0122-5, indexed in Pubmed: 26604987.

5. Colton T. Statistics in medicine. 1st ed. Little, Brown \& Company, Boston 1974.

6. Dugan SA, Bhat KP. Biomechanics and analysis of running gait. Phys Med Rehabil Clin N Am. 2005; 16(3): 603-621, doi: 10.1016/j.pmr.2005.02.007, indexed in Pubmed: 16005396.

7. Early JS, Bucholz RW. Lisfranc injuries and their management. Current Orthopaedics. 1996; 10(3): 169-173, doi: 10.1016/s0268-0890(96)90005-5.

8. Franco $\mathrm{AH}$. Pes cavus and pes planus. Analyses and treatment. Phys Ther. 1987; 67(5): 688-694, indexed in Pubmed: 3575426.

9. Fukano M, Fukubayashi T. Motion characteristics of the medial and lateral longitudinal arch during landing. Eur J Appl Physiol. 2009; 105(3): 387-392, doi: 10.1007/ s00421-008-0915-3, indexed in Pubmed: 18998155.

10. Glasoe WM, Yack HJ, Saltzman CL. Anatomy and Biomechanics of the First Ray. Physical Therapy. 1999; 79(9): 854-859, doi: 10.1093/ptj/79.9.854.

11. Hillstrom HJ, Song J, Kraszewski AP, et al. Foot type biomechanics part 1: structure and function of the asymptomatic foot. Gait Posture. 2013; 37(3): 445-451, doi: 10.1016/j.gaitpost.2012.09.007, indexed in Pubmed: 23107625. 
12. Kapandji I. The physiology of the joints. Longman Group Limited, Churchill Livingstone., London 1970.

13. Lautzenheiser SG, Kramer PA. Linear and angular measurements of the foot of modern humans: a test of Morton's foot types. Anat Rec (Hoboken). 2013; 296(10): 1526-1533, doi: 10.1002/ar.22764, indexed in Pubmed: 23963833.

14. Lundgren $P$, Nester $C$, Liu $A$, et al. Invasive in vivo measurement of rear-, mid- and forefoot motion during walking. Gait Posture. 2008; 28(1): 93-100, doi: 10.1016/j. gaitpost.2007.10.009, indexed in Pubmed: 18096389.

15. Lung CW, Yang SW, Hsieh LF. Is the Arch Index Meaningful. Korean Journal of Sport Biomechanics. 2009; 19(2): 187-196, doi: 10.5103/kjsb.2009.19.2.187.

16. Menz H, Munteanu S. Validity of 3 Clinical Techniques for the Measurement of Static Foot Posture in Older People. J Orthopaedic Sports Physical Therapy. 2005; 35(8): 479-486, doi: 10.2519/jospt.2005.35.8.479.

17. Moore KL, Dalley AF, Agur AMR. Clinically Oriented Anatomy. Wolters Kluwer Health 2013.

18. Murley GS, Menz HB, Landorf KB. A protocol for classifying normal- and flat-arched foot posture for research studies using clinical and radiographic measurements. J Foot Ankle Res. 2009; 2: 22, doi: 10.1186/1757-1146-2-22, indexed in Pubmed: 19575811.

19. Nester CJ, Jarvis HL, Jones RK, et al. Movement of the human foot in 100 pain free individuals aged 18-45: implications for understanding normal foot function. J Foot Ankle Res. 2014; 7(1): 51, doi: 10.1186/s13047014-0051-8, indexed in Pubmed: 25493100.

20. Nester CJ, Liu AM, Ward E, et al. In vitro study of foot kinematics using a dynamic walking cadaver model. J Biomech. 2007; 40(9): 1927-1937, doi: 10.1016/j. jbiomech.2006.09.008, indexed in Pubmed: 17081548.

21. Oatis CA. Biomechanics of the foot and ankle under static conditions. Phys Ther. 1988; 68(12): 1815-1821, indexed in Pubmed: 3057518.

22. Philbin T, Rosenberg G, Sferra JJ. Complications of missed or untreated Lisfranc injuries. Foot Ankle Clin. 2003; 8(1): 61-71, doi: 10.1016/s1083-7515(03)00003-2, indexed in Pubmed: 12760575 .
23. Pohl MB, Farr L. A comparison of foot arch measurement reliability using both digital photography and calliper methods. J Foot Ankle Res. 2010; 3: 14, doi: 10.1186/17571146-3-14, indexed in Pubmed: 20630090.

24. Roth S, Roth A, Jotanovic Z, et al. Navicular index for differentiation of flatfoot from normal foot. Int Orthop. 2013; 37(6): 1107-1112, doi: 10.1007/s00264-013-18856, indexed in Pubmed: 23580031.

25. Saltzman CL, Nawoczenski DA, Talbot KD. Measurement of the medial longitudinal arch. Arch Phys Med Rehabil. 1995; 76(1): 45-49, doi: 10.1016/50003-9993(95)800417, indexed in Pubmed: 7811174.

26. Saltzman CL, Nawoczenski DA. Complexities of foot architecture as a base of support. J Orthop Sports Phys Ther. 1995; 21(6): 354-360, doi: 10.2519/jospt.1995.21.6.354, indexed in Pubmed: 7655479.

27. Seringe $R$, Wicart $P$. The talonavicular and subtalar joints: the „calcaneopedal unit" concept. Orthop Traumatol Surg Res. 2013; 99(6 Suppl): S345-S355, doi: 10.1016/j. otsr.2013.07.003, indexed in Pubmed: 23978710.

28. Sinacore DR, Gutekunst DJ, Hastings MK, et al. Neuropathic midfoot deformity: associations with ankle and subtalar joint motion. J Foot Ankle Res. 2013; 6(1): 11, doi: 10.1186/1757-1146-6-11, indexed in Pubmed: 23531372.

29. Takai S. Structural components of the arch of the foot analyzed by radiogrammetric and multivariate statistical methods. Cells Tissues Organs. 1984; 119(3): 161-164, doi: 10.1159/000145879.

30. Viladot A. Biomechanics of the subtalar joint. Foot. 1992; 2(2): 83-88, doi: 10.1016/0958-2592(92)90023-i.

31. Wicart P. Cavus foot, from neonates to adolescents. Orthop Traumatol Surg Res. 2012; 98(7): 813-828, doi: 10.1016/j. otsr.2012.09.003, indexed in Pubmed: 23098772.

32. Yalçin N, Esen E, Kanatli U, et al. Evaluation of the medial longitudinal arch: a comparison between the dynamic plantar pressure measurement system and radiographic analysis. Acta Orthop Traumatol Turc. 2010; 44(3): 241-245, doi: 10.3944/AOTT.2010.2233, indexed in Pubmed: 21088466. 